\title{
Impacto do Uso de Dieta Imunomoduladora em Pacientes com Câncer Colorretal Submetidos a Cirurgias Eletivas com Abreviação de Jejum Pré-operatório
} Impact of the Use of Immunomodulatory Diet in Colorectal Cancer Patients under Elective Surgeries with Preoperative Fasting Abbreviation Impacto del Uso de la Dieta Inmunomoduladora en Pacientes con Cáncer Colorrectal Sometidos a Cirugía Electiva con Abreviatura de Ayuno Preoperatorio

\author{
Mariana Vieira Barbosa ${ }^{1}$; Flávia Mauro de Queiroz ${ }^{2}$; Nivaldo Barroso de Pinho ${ }^{3}$; Renata Brum Martucci ${ }^{4}$
}

\begin{abstract}
Resumo
Introdução: A função imune é geralmente suprimida por procedimentos cirúrgicos de grande porte e pela desnutrição proteico-energética. A imunomodulação se torna uma opção de terapêutica para pacientes com câncer submetidos a cirurgias, reduzindo complicações infecciosas no pós-operatório e tempo de internação hospitalar. Objetivo: Avaliar o impacto do uso de dieta imunomoduladora em pacientes com câncer colorretal submetidos a cirurgias eletivas com abreviação de jejum pré-operatório. Método: Foi realizada uma coorte de dados retrospectivos com pacientes com câncer colorretal submetidos à cirurgia no Instituto Nacional de Câncer José Alencar Gomes da Silva, em 2013. A amostra foi dividida em grupo 1 - pacientes que realizaram abreviação de jejum pré-operatório e receberam suplementação nutricional com dieta imunomoduladora no pré-operatório $(n=20)$; e grupo 2 - pacientes que realizaram apenas a abreviação de jejum pré-operatório $(n=30)$. Foram coletados dados de identificaçấo do paciente, clínicos e cirúrgicos dos prontuários. As análises estatísticas foram realizadas utilizando o programa SPSS, 17.0. Resultados: A amostra foi composta por 50 pacientes, submetidos à ressecção anterior do reto, com idade média de 61,9 anos $\pm 13,8$ anos, sendo $52 \%$ do sexo masculino. O sítio tumoral mais prevalente foi o reto (44\%). Não foram observadas diferenças significativas entre os grupos em relação às características nutricionais, clínicas e cirúrgicas, aos exames bioquímicos (pré e pós-operatórios), às intercorrências gastrointestinais, à ocorrência de complicaçóes no pós-operatório e à permanência hospitalar. Conclusão: Nas condiçôes estudadas, a imunomodulação no pré-operatório não contribuiu para redução de complicaçóes pós-operatórias, da incidência de intercorrências gastrointestinais e do tempo de internação hospitalar. Palavras-chave: Neoplasias Colorretais/cirurgia; Jejum; Imunomodulação; Cuidados Pré-operatórios
\end{abstract}

\footnotetext{
${ }^{1}$ Nutricionista. Residente em Nutriçáo Oncológica do Programa de Residência Multiprofissional em Oncologia do Instituto Nacional de Câncer José Alencar Gomes da Silva (INCA). Rio de Janeiro (RJ), Brasil. E-mail: marivieira89@gmail.com.

${ }^{2}$ Nutricionista. Especialista em Nutrição Oncológica pelo INCA. Nutricionista do Serviço de Nutrição e Dietética do Hospital do Câncer I (HCI) do INCA. Rio de Janeiro (RJ), Brasil.E-mail: flavia.queiroz@inca.gov.br.

${ }^{3}$ Nutricionista. Mestre em Nutrição Humana pela Universidade Federal do Rio de Janeiro (UFRJ). Doutorando em Nutrição Humana pela UFRJ. Chefe do Serviço de Nutrição e Dietética do HCI do INCA. E-mail: npinho@inca.gov.br.

${ }^{4}$ Nutricionista. Doutora em Ciências pela Universidade UFRJ. Pós-doutoranda do Programa de Epidemiologia Clínica da Pós-Graduaçấo em Oncologia do INCA. Nutricionista do Serviço de Nutrição e Dietética do HCI do INCA. Professor-Adjunto do Instituto de Nutriçấo da Universidade do Estado do Rio de Janeiro (UERJ). Rio de Janeiro (RJ), Brasil. E-mail: renata.martucci@inca.gov.br.

Endereço para correspondência: Mariana Vieira Barbosa. Rua Benjamin Constant, 134/409 - Glória. Rio de Janeiro (RJ), Brasil. CEP: 20.241-150.

E-mail: marivieira89@gmail.com.
} 


\section{INTRODUÇÃO}

O câncer é uma doença crônico-degenerativa considerada um problema de saúde pública mundial por sua elevada incidência, prevalência e mortalidade ${ }^{1}$. Os diagnósticos mais ocorridos, em 2012, foram os de câncer de pulmão (1,8 milhôes), mama (1,7 milhôes) e colorretal (1,4 milhôes). Este último foi o terceiro tipo de neoplasia mais comum entre os homens (746 mil casos - 10\% do total) e o segundo mais comum entre as mulheres (614 mil casos $-9,2 \%$ do total $)^{2}$.

No Brasil, foram estimados 576 mil casos novos de câncer para 2014; e, com exceção do câncer de pele não melanoma, o de cólon e reto aparece como o terceiro mais incidente, com estimativas de 32.600 novos casos e 14.016 mortes pela doença ${ }^{3}$. Mais de $90 \%$ dessa neoplasia acomete indivíduos com mais de 50 anos de idade, apresentando desenvolvimento relativamente lento e uma taxa de sobrevida, em cinco anos, de até $80 \%$ nos estágios iniciais e $5 \%$ nos estágios mais avançados ${ }^{4,5}$. A cirurgia é o tratamento mais frequente do câncer colorretal ${ }^{6}$.

O jejum pré-operatório estabelecido para a realização de procedimentos cirúrgicos tem sido bastante discutido atualmente. Os efeitos negativos da prática tradicional (jejum de 8-12 horas) estão bem estabelecidos, provocando desconforto como sede, ansiedade e dores de cabeça e intensificando o estresse metabólico ocasionado pelo trauma cirúrgico, o que leva a um aumento da resistência insulínica e a ocorrência de reações catabólicas no organismo ${ }^{7-9}$.

A abreviação do jejum para 2 horas antes da indução anestésica com líquidos claros é recomendada para a maioria dos pacientes candidatos a cirurgias abdominais eletivas, o que melhora a resistência insulínica e a função imune, e reduz o catabolismo proteico e as taxas de infecção pós-cirúrgicas, contribuindo para melhor desfecho ${ }^{9,10}$.

A função imune é geralmente suprimida por procedimentos cirúrgicos de grande porte, levando ao aumento da morbidade (principalmente as infecciosas), da mortalidade e da duração da permanência hospitalar. Além disso, a desnutrição proteico-energética, que ocorre em 30-90\% dos pacientes com câncer, também provoca efeitos adversos no sistema imunológico. A imunonutrição se torna uma opção de abordagem terapêutica para esses pacientes, reduzindo a incidência de complicaçôes pós-operatórias, já que modula o sistema imune e a resposta inflamatória do organismo ${ }^{11-13}$.

As dietas imunomoduladoras incluem glutamina, arginina, ácidos graxos ômega-3 e nucleotídeos (imunonutrientes), isolados ou combinados, e melhoram os mecanismos de defesa após cirurgias de grande porte, sendo utilizadas geralmente no período pré-operatório ${ }^{11,12,14}$.

Taxas de complicações pós-cirúrgicas em pacientes com câncer gastrointestinal, câncer de cabeça e pescoço e doenças cardíacas sugerem variações entre $15 \%$ e 54\%, estando inclusos complicaçōes infecciosas, infecçôes da ferida operatória, abcesso abdominal, pneumonia, infecçōes do trato urinário e sepse. Metanálises têm demonstrado redução significativa desses tipos de complicaçóes e do tempo de internação hospitalar em pacientes submetidos a cirurgias gastrointestinais com a utilização da terapia imunomoduladora nos pré, peri ou pós-operatórios ${ }^{15-18}$.

Segundo o guidelines da European Society for Parenteral and Enteral Nutrition (ESPEN), o uso de fórmulas imunomoduladoras em cirurgias eletivas do trato gastrointestinal alto tem nível de evidência $\mathrm{A}^{19}$. E, de acordo com o Consenso Nacional de Nutrição Oncológica ${ }^{20}$, há benefícios comprovados no uso de dietas imunomoduladoras em pacientes no pré-operatório de cirurgias eletivas oncológicas de grande porte.

Diante disso, o presente estudo teve como objetivo avaliar o impacto do uso de dieta imunomoduladora em pacientes com câncer colorretal submetidos a cirurgias eletivas com abreviação de jejum pré-operatório.

\section{MÉTODO}

Foi realizado um estudo de coorte, com coleta de dados retrospectivos, em pacientes com diagnóstico de câncer colorretal submetidos a procedimento cirúrgico para ressecção de tumor no Hospital do Câncer I do Instituto Nacional de Câncer José Alencar Gomes da Silva (HCI/ INCA), no ano de 2013.

Foram incluídos indivíduos de ambos os sexos, com idade igual ou superior a 20 anos, que realizaram abreviação de jejum pré-operatório, e que foram suplementados com dieta imunomoduladora no pré-operatório (no segundo semestre de 2013). Excluíram-se pacientes com restrição de sacarose ou diabéticos, com outro diagnóstico de câncer nos cinco anos anteriores, portadores de doenças inflamatórias crônicas e aqueles com falta de informaçóes nos prontuários necessárias ao estudo.

Em janeiro de 2013, foi implementada a abreviação de jejum pré-operatório no setor de Cirurgia Abdominopélvica do HCI pelo Serviço de Nutrição e Dietética. A abreviação de jejum é realizada com a administração de $237 \mathrm{ml}$ de mistura isenta de lipídeos, contendo carboidratos e proteínas, até 2 horas antes da cirurgia.

A partir de junho do mesmo ano, iniciou-se a terapia nutricional pré-operatória com dieta imunomoduladora, nutricionalmente completa, adicionada de arginina, 
nucleotídeos e ácidos graxos ômega-3, sendo administrada duas vezes ao dia ( $400 \mathrm{ml} / \mathrm{dia})$, durante cinco a sete dias antes do procedimento cirúrgico. Os pacientes foram convocados pelo ambulatório de nutrição uma semana antes do procedimento cirúrgico para receberem o suplemento imunomodulador.

A amostra foi dividida em dois grupos: o grupo 1 $(n=20)$ foi formado por pacientes que realizaram abreviação de jejum pré-operatório e receberam suplementação nutricional oral com dieta imunomoduladora, sendo submetidos à cirurgia no $2^{\circ}$ semestre de 2013 . O grupo $2(\mathrm{n}=30)$ foi formado por pacientes que realizaram apenas a abreviação de jejum pré-operatório e foram submetidos à cirurgia no $1^{\circ}$ semestre de 2013.

Os dados pessoais e clínicos dos pacientes foram obtidos por meio dos prontuários localizados no arquivo médico do HCI/INCA, registrados em ficha de coleta de dados própria e posteriormente inseridos em um banco de dados. Foram coletadas informaçóes referentes à identificação do paciente, à data de nascimento, à idade, ao sexo, à raça, à data da internação, e aos hábitos e estilo de vida, como tabagismo e etilismo.

Dados da história clínica e do procedimento cirúrgico foram obtidos dos registros médicos, a saber: tipo, localização e estadiamento do câncer, presença e localizaçáo de metástases, doenças associadas, tratamentos neoadjuvantes - quimioterapia e/ou radioterapia, tipo de cirurgia, via de acesso cirúrgico, horário de início e término do procedimento, tipo e volume de hidratação perioperatória e dia da alta hospitalar; bem como dados de mobilização pós-operatória e de ocorrência de complicações pós-cirúrgicas: infecções hospitalares, deiscência de anastomose, aparecimento de fístula, íleo, sepse e outras. Essas foram divididas em precoces (até sete dias do pós-operatório) ou tardias (até 30 dias do pós-operatório).

Os tempos de jejum pré e pós-operatórios, o tipo da dieta recebida pelo paciente no pós-operatório, sua via de administração, função intestinal e a presença de intercorrências durante um período de sete dias no pós-operatório (náuseas, vômitos, dor e distensão abdominal, diarreia etc.) foram obtidos pelos registros da nutrição. A classificação do estado nutricional pré-operatório foi obtida por meio da avaliação subjetiva global produzida pelo próprio paciente (ASG-PPP), instrumento de triagem nutricional aplicado em todos os pacientes nas primeiras 24 horas após a internação, atribuindo os escores A (bem nutrido), B (moderadamente desnutrido ou suspeita de desnutrição) e C (gravemente desnutrido), bem como pelo índice de massa corporal (IMC), segundo os padróes da Organização Mundial da Saúde ${ }^{21}$ para adultos, e da Organização Pan-Americana de Saúde ${ }^{22}$ para idosos.
Em relação aos dados bioquímicos, foram obtidos por meio de prontuário eletrônico os níveis séricos de glicose, proteínas totais, albumina e proteína $\mathrm{C}$ reativa (PCR), além de hemograma, contagem de plaquetas, leucócitos e neutrófilos, nos pré e pós-operatórios. Os resultados foram comparados com os padróes de referência do laboratório do HCI/INCA.

A coleta de dados foi realizada após a aprovação do Comitê de Ética em Pesquisa do INCA, sob protocolo $\mathrm{n}^{\circ}$ 658.874/14. Todos os procedimentos foram seguidos de acordo com as diretrizes e normas regulamentadoras de pesquisas envolvendo seres humanos (Resolução CNS $n^{\circ} 466 / 2012$ ).

Os dados obtidos foram tabulados em banco de dados no Excel $\left(\right.$ Microsoft $\left.^{\circ}\right)$ e analisados no programa SPSS, versão 17.0 (SPSS for Windows, 2004). Foi realizado teste de normalidade Kolmogorov-Smirnov, considerando variáveis normais ou paramétricas quando valores de p>0,05.

As variáveis categóricas foram apresentadas como frequência e percentual. Foi utilizado o teste Qui-quadrado ou teste exato de Fischer, quando necessário. Os resultados de variáveis contínuas foram expressos em média \pm desvio- padrão. As variáveis paramétricas foram comparadas entre os grupos do estudo pelos testes $t$ de Student não pareado e ANOVA, com um intervalo de confiança de $95 \%$, sendo estatisticamente significativo quando $\mathrm{p}<0,05$. No caso das variáveis não paramétricas, os testes de Mann-Whitney e Kruskal-Wallis foram utilizados para comparação entre os grupos, com um intervalo de confiança de $95 \%$, sendo estatisticamente significativos quando $\mathrm{p}<0,05$. $\mathrm{O}$ coeficiente de Pearson será obtido no teste de correlação entre as variáveis contínuas.

\section{RESULTADOS}

A amostra foi composta por 50 pacientes, com idade média de 61,9 anos, sendo $52 \%$ do sexo masculino e $48 \%$ do sexo feminino. Em relação à cor da pele, $50 \%$ eram brancos, 32\% eram pardos e $9 \%$ eram negros. Quanto ao estilo de vida, $38 \%$ dos pacientes eram tabagistas ou ex-tabagistas e $26 \%$ eram etilistas ou ex-etilistas.

$\mathrm{O}$ estado nutricional pré-operatório da amostra, avaliado pela ASG-PPP, classificou 48\% dos pacientes como bem nutridos (ASG A), 50\% como moderadamente desnutridos ou com suspeita de desnutrição (ASG B) e $2 \%$ como gravemente desnutridos (ASG C). O estado nutricional avaliado pelo IMC está demonstrado na Figura 1.

Em relação ao estadiamento da doença, 24\% tinham doença estágio I, assim como doença estágio II (24\%), $20 \%$ tinham doença estágio III, 14\% estágio IV e 18\% dos pacientes apresentavam estadiamento desconhecido. 
O sítio tumoral mais prevalente foi reto. A ressecção anterior do reto (RAR) foi a cirurgia mais realizada (Figura 1). Apenas sete pacientes apresentavam metástase a distância no momento do diagnóstico; sendo, desses, cinco no fígado, um no tórax e um em linfonodo mesentérico.
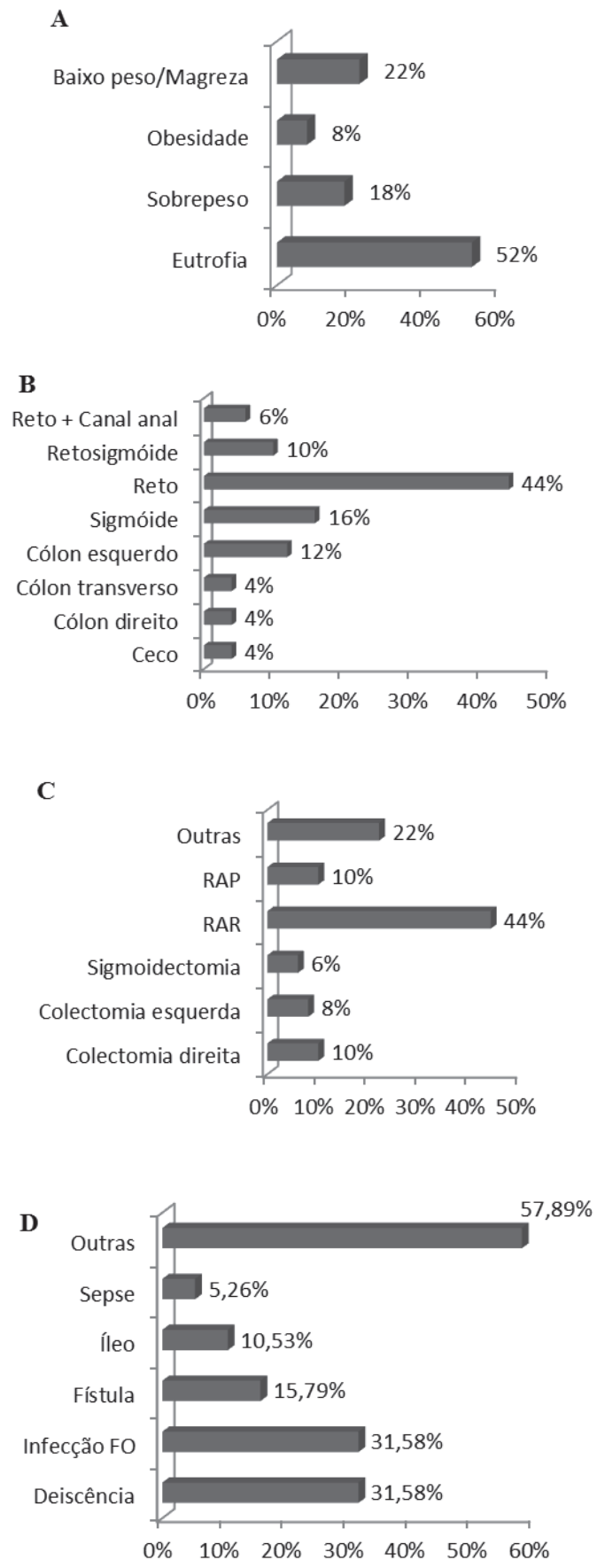

Figura 1. A. Distribuição percentual da amostra de acordo com o estado nutricional segundo IMC. B. Distribuição percentual da amostra de acordo com o sítio tumoral. C. Distribuição percentual da amostra de acordo com o tipo de cirurgia. D. Distribuição percentual dos pacientes $(n=19)$ de acordo com complicações pós-operatórias

$R A R=$ ressecção anterior do reto. $R A P=$ ressecção abdominopélvica.
Quanto à via de acesso cirúrgico, $48 \%$ das cirurgias foram por laparotomia, $44 \%$ por videolaparoscopia e $8 \%$ por robótica. Realizaram quimioterapia combinada com radioterapia neoadjuvantes $42 \%$ dos pacientes e $2 \%$ realizaram apenas quimioterapia; enquanto $56 \%$ dos pacientes não necessitaram de tratamento neoadjuvante.

$\mathrm{Na}$ comparação entre os grupos 1 e 2, não foram observadas diferenças significativas entre eles em relação às características nutricionais e cirúrgicas, conforme demonstrado na Tabela 1.

A média do tempo de jejum pré-operatório do total da amostra foi de $5 \mathrm{~h} 11 \mathrm{~min} 36 \mathrm{seg}$. Tiveram o tempo de jejum abaixo dessa média 27 pacientes (54\%), e apenas $11(22 \%)$ realizaram a abreviaçáo de jejum pré-operatório conforme preconizado (2 horas). Quanto ao tempo de jejum pós-operatório, a média foi de 27 h $04 \mathrm{~min} 42 \mathrm{seg}$, tendo 34 pacientes (68\%) iniciado a alimentação em até 24 horas após a cirurgia, com dieta líquida sem resíduos.

A Tabela 2 mostra as médias e desvios-padrão dos exames bioquímicos dos pacientes nos pré e pós-operatórios. Os valores de glicemia e hemoglobina foram os únicos que não tiveram diferença estatisticamente significativa entre os dois momentos. Os resultados encontrados são os esperados após um procedimento cirúrgico, como o aumento de proteínas de fase aguda positiva (PCR), leucócitos e neutrófilos e a redução de hemácias e hematócrito, em virtude do estresse metabólico e sangramento que ocorrem na cirurgia. Quando comparados os exames bioquímicos do grupo 1 com o grupo 2, tanto no pré quanto no pós-operatório, não foram observadas diferenças estatisticamente significativas.

Do total da amostra, 19 pacientes (38\%) tiveram pelo menos uma complicação pós-operatória; desses, $31,6 \%$ apresentaram infecções, como pode ser visto na Figura 1. Outras complicações apresentadas foram peritonite, acidose metabólica, hemorragia digestiva alta, prolapso de colostomia etc. Em relação à classificação das complicaçóes, $48,3 \%$ foram classificadas como precoces e 51,7\% como tardias. A Tabela 3 mostra a distribuição dos pacientes que tiveram complicaçóes pós-operatórias de acordo com a via de acesso cirúrgico e a cirurgia realizada, embora não tenha sido encontrada diferença estatística na taxa de complicaçóes entre os pacientes que realizaram cirurgia de grande porte (laparotomia exploradora) e as demais (videolaparoscopia e cirurgia robótica).

Não houve diferença significativa entre os dois grupos em relação à ocorrência de complicaçôes pós-cirúrgicas. Quanto às infecçôes pós-operatórias, um paciente (5\%) do grupo 1 (que recebeu suplementação com dieta imunomoduladora) manifestou essa complicação; enquanto, no grupo 2 , foram cinco pacientes $(16,7 \%)$; 
Tabela 1. Média e desvio-padrão das características dos grupos $1(n=20)$ e $2(n=30)$ e do total da amostra

\begin{tabular}{|c|c|c|c|c|}
\hline Variáveis & Total & Grupo 1 & Grupo 2 & P valor* \\
\hline Idade (anos) & $61,92 \pm 13,82$ & $60,85 \pm 14,38$ & $62,63 \pm 13,64$ & 0,660 \\
\hline Peso atual $(\mathrm{Kg})$ & $64,67 \pm 10,73$ & $65,42 \pm 11,57$ & $64,16 \pm 10,30$ & 0,689 \\
\hline Altura (m) & $1,62 \pm 0,10$ & $1,64 \pm 0,11$ & $1,61 \pm 0,10$ & 0,321 \\
\hline IMC (Kg/m²) & $24,69 \pm 4,28$ & $24,43 \pm 4,58$ & $24,87 \pm 2: 18: 04$ & 0,728 \\
\hline Tempo cirúrgico & $5: 28: 30 \pm 2: 14: 30$ & $6: 10: 15 \pm 2: 00: 26$ & $5: 00: 40 \pm 13,64$ & 0,073 \\
\hline Tempo jejum pré-operatório & $5: 11: 36 \pm 3: 29: 34$ & 4:18:15 $\pm 2: 59: 09$ & $5: 47: 10 \pm 3: 43: 24$ & 0,143 \\
\hline Tempo jejum pós-operatório & $\begin{array}{c}27: 04: 42 \pm \\
22: 48: 34\end{array}$ & $\begin{array}{c}33: 43: 30 \pm \\
33: 10: 35\end{array}$ & $\begin{array}{c}22: 38: 50 \pm \\
10: 21: 08\end{array}$ & 0,093 \\
\hline Tempo de internação (dias) & $10,12 \pm 8,20$ & $11,95 \pm 11,64$ & $8,90 \pm 4,57$ & 0,200 \\
\hline
\end{tabular}

*Diferença estatística entre os grupos 1 e 2 . Teste $t$ de Student náo pareado. IMC=índice de massa corporal.

Tabela 2. Comparação entre os exames bioquímicos nos pré e pós-operatórios do total da amostra

\begin{tabular}{l|c|c|c|c}
\multicolumn{1}{c|}{ Exames } & N & Pré-operatório & Pós-operatório & P valor \\
\hline Glicemia $(\mathrm{mg} / \mathrm{dL})$ & 50 & $87,40 \pm 39,14$ & $79,68 \pm 54,45$ & 0,410 \\
\hline Proteínas totais $(\mathrm{g} / \mathrm{dL})$ & 50 & $6,59 \pm 8,98$ & $2,16 \pm 1,92$ & 0,001 \\
\hline Albumina $(\mathrm{g} / \mathrm{dL})$ & 43 & $4,07 \pm 0,60$ & $1,55 \pm 0,92$ & 0,000 \\
\hline PCR $(\mathrm{mg} / \mathrm{dL})$ & 40 & $0,86 \pm 1,27$ & $12,08 \pm 7,66$ & $0,000^{*}$ \\
\hline Hemácias $(\mathrm{em} \mathrm{milhões/ \mu )}$ & 50 & $4,33 \pm 0,57$ & $3,82 \pm 0,54$ & 0,000 \\
\hline Hemoglobina $(\mathrm{mg} / \mathrm{dL})$ & 50 & $12,17 \pm 2,29$ & $12,55 \pm 13,18$ & 0,843 \\
\hline Hematócrito $(\%)$ & 50 & $37,64 \pm 6,14$ & $32,91 \pm 5,04$ & 0,000 \\
\hline Plaquetas $(\mathrm{k} / \mu \mathrm{L})$ & 50 & $283.900 \pm 112.460$ & $240.500 \pm 97.806$ & $0,000^{*}$ \\
\hline Leucócitos $(\mu \mathrm{L})$ & 50 & $6.101 \pm 2.140$ & $12.909 \pm 13.781$ & $0,001^{*}$ \\
\hline Neutrófilos $(/ \mu \mathrm{L})$ & 50 & $3.778 \pm 1.652$ & $9.319 \pm 3.757$ & 0,000 \\
\hline
\end{tabular}

Teste $t$ de Student não pareado (paramétricos) e *Mann-whitney (não paramétrico). PCR=proteína C reativa.

Tabela 3. Número absoluto de pacientes que apresentaram e que não apresentaram complicações pós-operatórias de acordo com a via de acesso cirúrgico e cirurgia realizada

\begin{tabular}{|c|c|c|c|c|}
\hline & & \multicolumn{3}{|c|}{ Complicação pós-operatória } \\
\hline & & Sim & Não & Total \\
\hline \multirow{4}{*}{ Via de acesso cirúrgico } & Laparotomia exploradora & 9 & 15 & 24 \\
\hline & Videolaparoscopia & 8 & 14 & 22 \\
\hline & Robótica & 2 & 2 & 4 \\
\hline & Total & 19 & 31 & 50 \\
\hline \multirow{17}{*}{ Cirurgia } & RAR & 6 & 16 & 22 \\
\hline & RAP & 3 & 2 & 5 \\
\hline & Colectomia direita & 1 & 4 & 5 \\
\hline & Colectomia esquerda & 1 & 3 & 4 \\
\hline & Sigmoidectomia & 1 & 2 & 3 \\
\hline & Ileocolectomia direita & 1 & 0 & 1 \\
\hline & Sigmoidectomia e hepatectomia direita & 0 & 1 & 1 \\
\hline & Sigmoidectomia, enterectomia e cistectomia & 1 & 0 & 1 \\
\hline & Sigmoidectomia, HTA, SOB e colecistectomia & 0 & 1 & 1 \\
\hline & RAP, HTA e SOB & 1 & 0 & 1 \\
\hline & RAP, sacrectomia (S5) e colpectomia parcial & 1 & 0 & 1 \\
\hline & RAR e setorectomia hepática lateral esquerda & 1 & 0 & 1 \\
\hline & RAR e SOB & 0 & 1 & 1 \\
\hline & RAR, HTA e SOB & 1 & 0 & 1 \\
\hline & RAR e colectomia esquerda & 0 & 1 & 1 \\
\hline & RAR e EPT & 1 & 0 & 1 \\
\hline & Total & 19 & 31 & 50 \\
\hline
\end{tabular}

RAR=ressecção anterior do reto. RAP=ressecção abdominopélvica. $\mathrm{HTA}=$ histerectomia total abdominal. $\mathrm{SOB}=$ salpingo-oforectomia bilateral. EPT=exenteraçăo pélvica total. 
porém, essa diferença também não foi estatisticamente significativa. Desses pacientes que apresentaram infecção no pós-operatório, um evoluiu para sepse (grupo 2) e um para óbito (grupo 1), sendo o único paciente do estudo que teve esse desfecho durante a internação.

Dos pacientes do grupo 1,75\% iniciaram a dieta no $1^{\circ} \mathrm{DPO}$ (dia de pós-operatório), enquanto $76,7 \%$ dos pacientes do grupo 2 iniciaram a dieta também nesse dia, não havendo diferença significativa entre eles. A Tabela 4 mostra a frequência de intercorrências gastrointestinais apresentadas pelos pacientes durante os sete dias de pós-operatório, por grupo. As intercorrências mais incidentes foram náuseas, vômitos, dor e distensão abdominal. A primeira alta hospitalar ocorreu no $3^{\circ} \mathrm{DPO}$, e no $5^{\circ}$ $\mathrm{DPO}$, mais da metade dos pacientes já tinham recebido alta $(60 \%)$.

\section{DISCUSSÃO}

Desnutrição, doenças malignas e estresse cirúrgico podem prejudicar a funçấo imune dos pacientes. As infecçóes são um grande problema após grandes cirurgias abdominais, contribuindo para o aumento da morbidade, da mortalidade e da duração da permanência hospitalar. Suplementos imunomoduladores com componentes dietéticos, como: arginina, glutamina, ácidos graxos ômega-3 e nucleotídeos, ganham cada vez mais atenção por promoverem efeitos benéficos no sistema imune, além de seu valor nutricional, em comparação com fórmulas nutricionais padrão ${ }^{12,13,14}$.

Helminen, Raitanen e Kellosalo ${ }^{14}$, Gunerhan et al. ${ }^{11}$, Fujitani et al. ${ }^{13}$ avaliaram os efeitos da suplementação pré-operatória em pacientes com tumores gastrointestinais, candidatos a cirurgias eletivas. Os pacientes foram divididos em grupos nos estudos, e um deles recebeu de $900 \mathrm{ml} /$ dia a $1.000 \mathrm{ml} /$ dia de dieta imunomoduladora por cinco a sete dias consecutivos antes do procedimento cirúrgico. Em nenhum dos estudos foram encontradas diferenças estatisticamente significativas entre os grupos em relação às taxas de complicaçóes pós-operatórias e tempo de internação hospitalar.

No presente estudo, 20 pacientes receberam suplemento imunomodulador $(400 \mathrm{ml} / \mathrm{dia})$ por cinco a sete dias consecutivos antes do procedimento cirúrgico (grupo 1), enquanto 30 pacientes náo foram suplementados (grupo 2). Não houve diferença estaticamente significativa quanto às complicaçóes infecciosas pós-operatórias e tempo de permanência hospitalar, como nos estudos supracitados.

Contudo, em uma metanálise realizada por Osland et al. ${ }^{23}$, que incluiu 21 estudos que compararam o uso de dietas imunomoduladoras com dietas padrão em pacientes com malignidades gastrointestinais submetidos a cirurgias, foram encontradas diferenças significativas na redução de complicações infecciosas e tempo de internação quando a suplementaçáo ocorreu nos períodos peri ou pós-operatórios. A suplementação pré-operatória não mostrou vantagem sobre a nutrição padrão em nenhum desfecho clínico, evidenciando a importância do momento que deve ser administrada a dieta imunomoduladora nesses pacientes.

Outras metanálises também têm demonstrado importante redução de complicaçóes infecciosas e do tempo de internação hospitalar em pacientes submetidos a cirurgias gastrointestinais com a utilização da terapia imunomoduladora nos pré, peri ou pós-operatórios ${ }^{15-18}$; porém, Cerantola et al. ${ }^{16}$ citam que os estudos incluídos em sua metanálise mostraram heterogeneidade significante com respeito aos pacientes, grupos-controle e duraçáo da suplementaçáo com imunomodulador, limitando as análises de grupo.

Na literatura, há uma gama de estudos avaliando os efeitos da imunonutriçáo no desfecho clínico de pacientes submetidos a cirurgias do trato gastrointestinal. Contudo, pode-se observar que eles apresentam diferenças quanto ao tamanho da amostra, diagnóstico da doença, localização da cirurgia, idade e estado nutricional dos pacientes, momento de início e duração da suplementação, quantidade total diária de suplemento fornecido aos pacientes e composição

Tabela 4. Frequência das intercorrências apresentadas pelos grupos $1(n=20)$ e $2(n=30)$ do $1^{\circ}$ ao $7^{\circ}$ DPO

\begin{tabular}{l|c|c|c|c|c}
\hline \multicolumn{1}{c|}{ Grupo 1 } & \multicolumn{3}{c}{ Grupo 2 } \\
\hline \multicolumn{1}{c|}{ DPO } & Intercorrências & Total de pacientes & Intercorrências & Total de pacientes & P valor \\
\hline $1^{\circ} \mathrm{DPO}$ & $20,0 \%$ & 20 & $26,7 \%$ & 30 & $\mathrm{NS}$ \\
\hline $2^{\circ} \mathrm{DPO}$ & $30,0 \%$ & 20 & $46,7 \%$ & 30 & $\mathrm{NS}$ \\
\hline $3^{\circ} \mathrm{DPO}$ & $42,1 \%$ & 19 & $36,7 \%$ & 30 & $\mathrm{NS}$ \\
\hline $4^{\circ} \mathrm{DPO}$ & $50,0 \%$ & 12 & $43,8 \%$ & 16 & $\mathrm{NS}$ \\
\hline $5^{\circ} \mathrm{DPO}$ & $55,5 \%$ & 9 & $63,6 \%$ & 11 & $\mathrm{NS}$ \\
\hline $6^{\circ} \mathrm{DPO}$ & $37,5 \%$ & 8 & $50,0 \%$ & 8 & $\mathrm{NS}$ \\
\hline $7^{\circ} \mathrm{DPO}$ & $37,5 \%$ & 8 & $50,0 \%$ & 8 & $\mathrm{NS}$ \\
\hline
\end{tabular}

NS: não significativo. 
do suplemento, como ressaltado por Cerantola et al. ${ }^{16}$. Isso possivelmente corrobora os diferentes resultados encontrados nesses estudos, dificultando o entendimento de qual população se beneficiaria mais dessa terapêutica e em que momento.

O projeto ERAS (Enhanced Recovery After Surgery) recomenda a ingestão de líquidos claros 2 horas antes da indução anestésica e de sólidos 6 horas antes, tanto para as cirurgias eletivas de cólon quanto para as de reto ${ }^{24,25}$. Essa é uma das recomendaçôes desse Consenso, com a finalidade de reduzir complicaçóes cirúrgicas e sintomas gastrointestinais e acelerar a recuperação pós-operatória do paciente. No presente estudo, os pacientes ficaram em média 5 horas em jejum, até o momento do procedimento cirúrgico. Além disso, eles não consumiram sólidos no dia anterior da cirurgia, recebendo apenas líquidos sem resíduos.

Quanto ao pós-operatório, a média do tempo de jejum foi 27 horas. A recomendação do ERAS, para cirurgias de reto, é o retorno da alimentação 4 horas após o procedimento; e, para as cirurgias de cólon, é recomendado que os pacientes sejam encorajados a comer dieta normal o mais breve possível depois da cirurgia ${ }^{24,25}$. No presente estudo, $76 \%$ dos pacientes iniciaram a alimentação nas primeiras 24 horas após o procedimento, porém todos com dieta líquida restrita no $1^{\circ} \mathrm{DPO}$, que foi sendo evoluída conforme tolerância individual.

Para que a abreviação de jejum perioperatório possa ser eficaz, não se deve pensar apenas nos tempos de jejuns pré e pós-operatórios, mas em uma série de medidas que são recomendadas e devem ser adotadas para otimizar o cuidado dos pacientes com câncer colorretal submetidos a cirurgias, como a reduçáo da prática do preparo mecânico de cólon, a profilaxia de náuseas e vômitos pós-operatórios, a otimização do manejo de fluidos perioperatório, a mobilizaçáo precoce após o procedimento etc. A única medida adotada com os pacientes que participaram deste estudo foi a administração da mistura hipolipídica até 2 horas antes da cirurgia, demonstrando a necessidade de criação de um protocolo pela equipe multiprofissional para melhor desfecho dessa população.

O presente estudo teve algumas limitaçóes: número reduzido de pacientes; foram incluídas cirurgias realizadas por videolaparoscopia e robótica, menos invasivas e com menor risco de complicações no pós-operatório; os pacientes receberam os suplementos ambulatorialmente e foram orientados quanto à dose e forma de administração, porém não se teve controle se seguiram as orientaçôes e o grau de aceitação dos suplementos; a dose administrada foi de $400 \mathrm{ml} /$ dia de dieta imunomoduladora pré-operatória. Náo existem consenso e recomendações quanto à dose de administração, porém a maior parte dos estudos administra de 500 a $1.000 \mathrm{ml} /$ dia de imunomodulador ${ }^{12}$. Esses fatores podem ter contribuído para os resultados encontrados no estudo.

\section{CONCLUSÃO}

Nas condiçóes estudadas, o uso de dieta imunomoduladora no pré-operatório em pacientes com câncer colorretal submetidos a cirurgias eletivas, somado à abreviação de jejum pré-operatório, não contribuiu para redução de complicaçôes pós-operatórias, da incidência de intercorrências gastrointestinais e do tempo de internação hospitalar.

Nesse contexto, sugere-se a continuação do estudo, com objetivo de aumentar a amostra, abrangendo especificamente o pré-operatório de cirurgias abdominais de grande porte e um monitoramento adequado quanto à administração da dieta imunomoduladora.

\section{CONTRIBUIÇÕES}

Mariana Vieira Barbosa participou da concepçáo e planejamento do projeto de pesquisa; coleta, análise e interpretação dos dados; redação final do artigo; aprovação da versão a ser publicada. Flávia Mauro de Queiroz e Nivaldo Barroso de Pinho participaram da concepçáo e planejamento do projeto de pesquisa; aprovação da versão a ser publicada. Renata Brum Martucci participou da concepção e planejamento do projeto de pesquisa; análise e interpretação dos dados; revisão crítica do artigo; aprovação da versão a ser publicada.

\section{Declaraçáo de Conflito de Interesses: Nada a Declarar.}

\section{RERERÊNCIAS}

1. Herr GE, Kolankiewicz, ACB, Berlezi EM, Gomes JS, Magnano TSBS, Rosanelli CP, et al. Avaliação de conhecimentos acerca da doença oncológica e práticas de cuidado com a saúde. Rev Bras Cancerol. 2013;59(1):33-41.

2. International Agency for Research on Cancer. GLOBOCAN 2012: Estimated cancer incidence, mortality and prevalence worldwide in 2012 [Internet]. Lyon, France: IARC; 2013 [acesso 2014 fev 08]. Disponível em: http://globocan.iarc.fr/Pages/fact_ sheets_cancer.aspx.

3. Instituto Nacional de Câncer José Alencar Gomes da Silva. Estimativa 2014: incidência de câncer no Brasil. Rio de Janeiro: INCA; 2014.

4. Fortes RC, Recôva VL, Melo AL, Novaes MRCG. Hábitos dietéticos de pacientes com câncer colorretal em fase pós-operatória. Rev Bras Cancerol. 2007;53(3):277-89. 
5. Fey A, Teixeira JVC, Teixeira JVC, Schinko FB, Vieira AM, Becker I. Perfil epidemiológico e evolução dos pacientes com câncer do colon e reto atendidos no Hospital Regional Alto Vale no ano de 2008. ACM Arq Catarin Med. 2010;39(4):62-7.

6. Castro LS. Câncer de Reto. In: Castro LS, Corrêa JHS. Tratamento cirúrgico do câncer gastrointestinal. Rio de Janeiro; 2005. p. 361-416.

7. Faria MSM, Aguilar-Nascimento JE, Pimenta OS, Alvarenja Júnior LC, Dock-Nascimento DB, Slhessarenko N. Preoperative fasting of 2 hours minimizes insulin resistance and organic response to trauma after videocholecystectomy: a randomized, controlled, clinical trial. World J Surg. 2009;33(6):1158-64.

8. Perrone F, Silva Filho AC, Adôrno IF, Anabuki NT, Leal FS, Colombo T, et al. Effects of preoperative feeding with a whey protein plus carbohydrate drink on the acute phase response and insulin resistance. A randomized trial. Nutr J. 2011;10:66.

9. Viganò J, Cereda E, Caccialanza R, Carini R, Carmeletti B, Spampinato M, et al. Effects of preoperative oral carbohydrate supplementation on postoperative metabolic stress response of patients undergoing elective abdominal surgery. World J Surg. 2012 Aug;36(8):173843.

10. Dock-Nascimento DB, Aguilar-Nascimento JE, Faria MSM, Caporossi C, Slhessarenko N, Waitzberg DL. Evaluation of the effects of a preoperative 2-hour fast with maltodextrine and glutamine on insulin resistance, acute-phase response, nitrogen balance, and serum glutathione after laparoscopic cholecystectomy: a controlled randomized trial. JPEN J Parenter Enteral Nutr. 2012 Jan;36(1):43-52.

11. Gunerhan Y, Koskal N, Sahin UY, Uzun MA, EksiogluDemiralp E. Effect of preoperative immunonutrition and other nutrition models on cellular immune parameters. World J Gastroenterol. 2009 Jan;15(4):467-72.

12. Mizock BA, Sriram K. Perioperative immunonutrition. Expert Rev Clin Immunol. 2011 Jan;7(1):1-3.

13. Fujitani K, Tsujinaka T, Fujita J, Miyashiro I, Imamura $\mathrm{H}$, Kimura Y, et al. Prospective randomized trial of preoperative enteral immunonutrition followed by elective total gastrectomy for gastric câncer. Br J Surg. 2012;99(5):621-29.

14. Helminen H, Raitanen M, Kellosalo J. Immunonutrition in elective gastrointestinal surgery patients. Scand J Surg. 2007;96(1):46-50

15. Waitzberg DL, Saito H, Plank LD, Jamieson GG, Jagannath P, Hwang TL, et al. Postsurgical infections are reduced with specialized nutrition support. World J Surg. 2006 Aug;30(8):1592-604.

16. Cerantola Y, Hübner M, Grass F, Demartines N, Schäfer $\mathrm{M}$. Immunonutrition in gastrointestinal surgery. $\mathrm{Br} \mathrm{J}$ Surg. 2011 Jan;98(1):37-48.

17. Marimuthu K, Varadhan KK, Ljunggvist O, Lobo DN. A meta-analysis of the effect of combinations of immune modulating nutrients on outcome in patients undergoing major open gastrointestinal surgery. Ann Surg. 2012 Jun;255(6):1060-8.

18. Zhang Y, Gu Y, Guo T, Li Y, Cai H. Perioperative immunonutrition for gastrointestinal cancer: a systematic review of randomized controlled trials. Surg Oncol. 2012 Jun;21(2):e87-95.

19. Kreymann KG, Berger MM, Deutz NEP, Hiesmayr M, Jolliet P, Kazandjiev G, et al. ESPEN Guidelines on Enteral Nutrition: Intensive care. Clin Nutr. 2006 Apr;25(2):210-23.

20. Instituto Nacional de Câncer (BR). Consenso nacional de nutrição oncológica: volume 2. Rio de Janeiro: INCA; 2011.

21. Wold Health Organization. Physical status: the use and interpretation of anthropometry. Geneva: Wold Health Organization; 1995. WHO technical report series, 854.

22. Organización Panamericana de la Salud. XXXVI Reunión del Comité Asesor de Investigaciones en Salud: Encuestra multicêntrica: salud bienestar y envejecimiento.(SABE) en América Latina e el Caribe: informe preliminar [Internet]. [acesso em 2015 Ago. 29]. Disponível em: http://envejecimiento.csic.es/documentos/documentos/ paho-salud-01.pdf

23. Osland E, Hossain MB, Khan S, Memon MA. Effect of timing of pharmaconutrition (immunonutrition) administration on outcomes of elective surgery for gastrointestinal malignancies: a systematic review and meta-analysis. JPEN J Parenter Enteral Nutr. 2014 Jan;38(1):53-69.

24. Gustafsson UO, Scott MJ, Schwenk W, Demartines N, Roulin D, Francis N, et al.; Enhanced Recovery After Surgery Society. Guidelines for perioperative care in elective colonic surgery: Enhanced Recovery After Surgery (ERAS) Society recommendations. Clin Nutr. 2012 Dec;31(6):783-800.

25. Nygren J, Thacker J, Carli F, Fearon KCH, Norderval S, Lobo DN, et al.; Enhanced Recovery After Surgery Society. Guidelines for perioperative care in elective rectal/pelvic surgery: Enhanced Recovery After Surgery (ERAS) Society recommendations. Clin Nutr. 2012 Dec;31(6):801-16. 


\section{Abstract}

Introduction: The immune function is usually suppressed by major surgical procedures and protein-energy malnutrition. The immunomodulation becomes a therapeutic option for cancer patients undergoing surgery, reducing infectious complications after surgery, as well as hospital stay. Objective: To evaluate the impact of the use of an immunomodulatory diet in colorectal cancer patients undergoing elective surgeries with preoperative fasting abbreviation. Method: A cohort with retrospective data was performed with colorectal cancer patients who underwent surgery in the Brazilian National Cancer Institute José Alencar Gomes da Silva, in 2013. The sample was divided into two groups. Group 1: patients who underwent preoperative fasting abbreviation and who received nutritional supplementation with a preoperative immunomodulatory $\operatorname{diet}(\mathrm{n}=20)$; and group 2: patients who underwent only the abbreviation of fasting preoperatively $(n=0)$. The study collected patient identification data, together with clinical and surgical data from the records. Statistical analyzes were performed using SPSS, 17.0. Results: The sample consisted of 50 patients who underwent an anterior resection of the rectum, with a mean age of 61.9 years \pm 13.8 years; and $52 \%$ male. The most prevalent tumor site was the rectum (44\%). No significant differences were observed between the groups regarding nutritional, clinical and surgical characteristics, the biochemical tests (pre and postoperative), gastrointestinal complications, the occurrence of complications after surgery and hospital stay. Conclusion: Within the studied conditions, preoperative immunomodulation did not contribute to a reduction of postoperative complications, incidences of gastrointestinal complications and hospital stay.

Key words: Colorectal Neoplasms/surgery; Fasting; Immunomodulation; Preoperative Care

\section{Resumen}

Introducción: La función inmune generalmente se suprime por procedimientos quirúrgicos mayores y por la malnutrición proteico-energética. La inmunomodulación se convierte en una opción terapéutica para los pacientes con cáncer sometidos a cirugía mediante la reducción de las complicaciones infecciosas después de la cirugía y la estancia hospitalaria. Objetivo: Evaluar el impacto del uso de la dieta inmunomoduladora en pacientes con cáncer colorrectal sometidos a cirugía electiva con abreviatura ayuno preoperatorio. Método: A los datos retrospectivos de cohortes de pacientes con cáncer colorrectal que se sometió a una cirugía en el Instituto Nacional del Cáncer José Alencar Gomes da Silva se llevó a cabo en 2013. La muestra se dividió en el grupo 1: pacientes que se sometieron abreviatura ayuno preoperatorio y recibieron suplementos nutricionales con una dieta inmunomoduladora antes de la operación $(\mathrm{n}=20)$; y el grupo 2: pacientes que se sometieron a sólo abreviatura ayuno preoperatorio $(\mathrm{n}=30)$. Se recogieron los datos de identificación del paciente, clínica y quirúrgicos de los registros médicos. Los análisis estadísticos se realizaron con SPSS, 17.0. Resultados: La muestra estuvo constituida por 50 pacientes, que fueron sometidos a la que se sometieron a resección anterior rectal, con una edad media 61,9 años $\pm 13,8$ años, $52 \%$ varones, (44\%) No se observaron diferencias significativas entre los grupos en relación a las características nutricionales, clínicas y quirúrgicas, exámenes bioquímicos (pre y postoperatorios), a las complicaciones gastrointestinales, la aparición de complicaciones postoperatorias y la estancia hospitalaria. Conclusión: En las condiciones estudiadas, la inmunomodulación en el preoperatorio no contribuyó a la reducción de las complicaciones postoperatorias, la incidencia de complicaciones gastrointestinales y la estancia hospitalaria.

Palabras clave: Neoplasias Colorrectales/cirugía; Ayuno; Inmunomodulación; Cuidados Preoperatorios 